\title{
iPhone Independent Real Time Localization System Research and Its Healthcare Application
}

\author{
Xintong $\mathrm{Lu}^{1}$, Wei Liu ${ }^{2}$, Yongliang Guan ${ }^{1}$ \\ ${ }^{1}$ EEE, Nanyang Technological University, Singapore City, Singapore \\ ${ }^{2}$ Singapore Institute of Manufacturing Technology, Singapore City, Singapore \\ Email: xlu008@e.ntu.edu.sg,wliu@simtech.a-star.edu.sg,EYLGUAN@ntu.edu.sg \\ Received March 10, 2013; revised April 30, 2013; accepted May 15, 2013
}

Copyright (C) 2013 Xintong Lu et al. This is an open access article distributed under the Creative Commons Attribution License, which permits unrestricted use, distribution, and reproduction in any medium, provided the original work is properly cited.

\begin{abstract}
This project studied several popular localization algorithms on iPhone and, according to the demands, specifically designed it to improve healthcare IT system in hospitals. The challenge of this project was to realize the different localization systems on iPhone and to make balance between its response time and localization accuracy. We implemented three popular localization algorithms, namely nearest neighbor (NN), K-nearest neighbor (KNN), and probability phase, and we compared their performance on iPhone. Furthermore, we also implemented a real-time localization system using the ZigBee technology on iPhone. Thus, the whole system could realize not only self-localization but also others-localization. To fulfill the healthcare needs, we developed an application, which can be used to improve the hospital IT, system. The whole project included three phases. The first phase was to localize iPhone's position using the received WiFi signal by iPhone, compare and optimize their performances. During the second phase, we implemented a ZigBee RFID localization system and combined it with the WiFi system. Finally, we combined new features of the system with a healthcare IT system. We believe that this application on iPhone can be a useful and advanced application in hospitals.
\end{abstract}

Keywords: iPhone; RTLS; WiFi; Healthcare

\section{Introduction}

In healthcare industry, there is a trend to decrease the doctors' workload using advanced IT technology. With the development of technology, the healthcare technology in hospitals is more and more advanced and efficient. During daily ward round of doctor, it is quite inconvenient for doctors to check every patient's cases, especially if there are lots of patients in one hospital. Traditional way to cope with it was that doctors had to memorize all cases of different patients or brought case files with themselves. Nowadays, many hospitals have used ecommerce, which will import patient cases into hospital system or even national health care system to help doctors to check patient history cases easily. By connection with this system, we can also simplify the process of ward round of doctor by our localization system. Furthermore, tracking patients' location and behavior is also useful for doctors. According to recent statistics of Midland psychiatric ward, more than 1000 mentally ill patients have escaped from the wards in the last three years, which is proved that tracking patient is a significant technology in hospitals. Finally, this localization application can also supply a function of indoor navigation to patients. No one can deny that finding a right way in an unfamiliar hospital is a tough task, especially for patients. Hence, if a mobile application can guide them to right area, it will help them save time. In our application, we developed a sub-application for patients with iPhone compass module to realize indoor navigation.

Radio frequency identification (RFID) [1] is a widely used technology in bank business field, security field and short distance communication field. The basic components of RFID system are tags, readers and application software. RFID readers can detect and read the data, which is emitted from tags through a defined radio frequency and protocol. Tags can be divided into two types: passive tags and active tags. For passive tags, they will not emit a signal by themselves. Instead, they generate a response by the power of reader's signal. It has both pros and cons. Obviously, it is energy-free, thus enabling tags to be used in a long term and making tags portable. However, the transmission range is only about $10 \mathrm{~m}$ to $5 \mathrm{me}-$ ters. Alternatively, active RFID tags will transmit signal 
actively. They have a longer working range, which will arrive at tens of meters. In our ZigBee localization system, we use active tags. The reason of it is that not only long-range but also more accurate localization than passive tags can be achieved.

Localization system has many ways to be implemented, such as Global Positioning System (GPS), cellular (AGPS) and etc. GPS provides information of users' location and time anywhere on earth. This system contains 24 localization satellites. Thus it can provide a satisfied service outdoors. But this above way has a limitation, though they are efficiently used in outdoors, they cannot contribute to indoors localization accuracy. Nowadays, existing methods for indoor localization are LANDMARC, RADAR, Cricket Location Support System, Angle of arrival, Time of arrival and Fingerprinting algorithm [2,3]. In this paper, because of iPhone limitation, we choose fingerprinting algorithm utilizing WiFi signal. WiFi signal indoors localization is a popular technology. For the reason that more and more mobile devices contain WiFi function, there is no extra-device needed to realize self-localization.

This paper focuses on independent indoor localization system implemented on iPhone and performance comparison of different algorithms and its healthcare application. The advantage of independent localization system is that we needn't have to connect to an AP to access a server. That means even we don't know the password of the WiFi signals, we still can use them to do localization. Section 2 discusses the implementation procedure and two systems structure. Section 3 provides the algorithm explanation and performance analysis among different algorithms and different systems. Section 4 introduces the application about healthcare and method of implementation. Finally, Section 5 concludes the paper briefly.

\section{Implementation}

\subsection{Complete Structure of Our Prototype}

Figure 1 shows the complete structure of the doctor's application. As we can see from the flow diagram, there are three main functions in this application: self-localization, patient localization, ward round application. Because this application has to use WiFi module of iPhone, it is mandatory to turn on WiFi before the application operation. There are two localization systems in this application: WiFi localization system and ZigBee localization system.

\subsection{WiFi Localization System}

For the WiFi system, we use SDK Xcode to program an application in iPhone to scan WiFi signal periodically. In fact, obtaining and scanning RSSI values in iPhone is proved to be a tough task $[4,5]$. Because Apple company

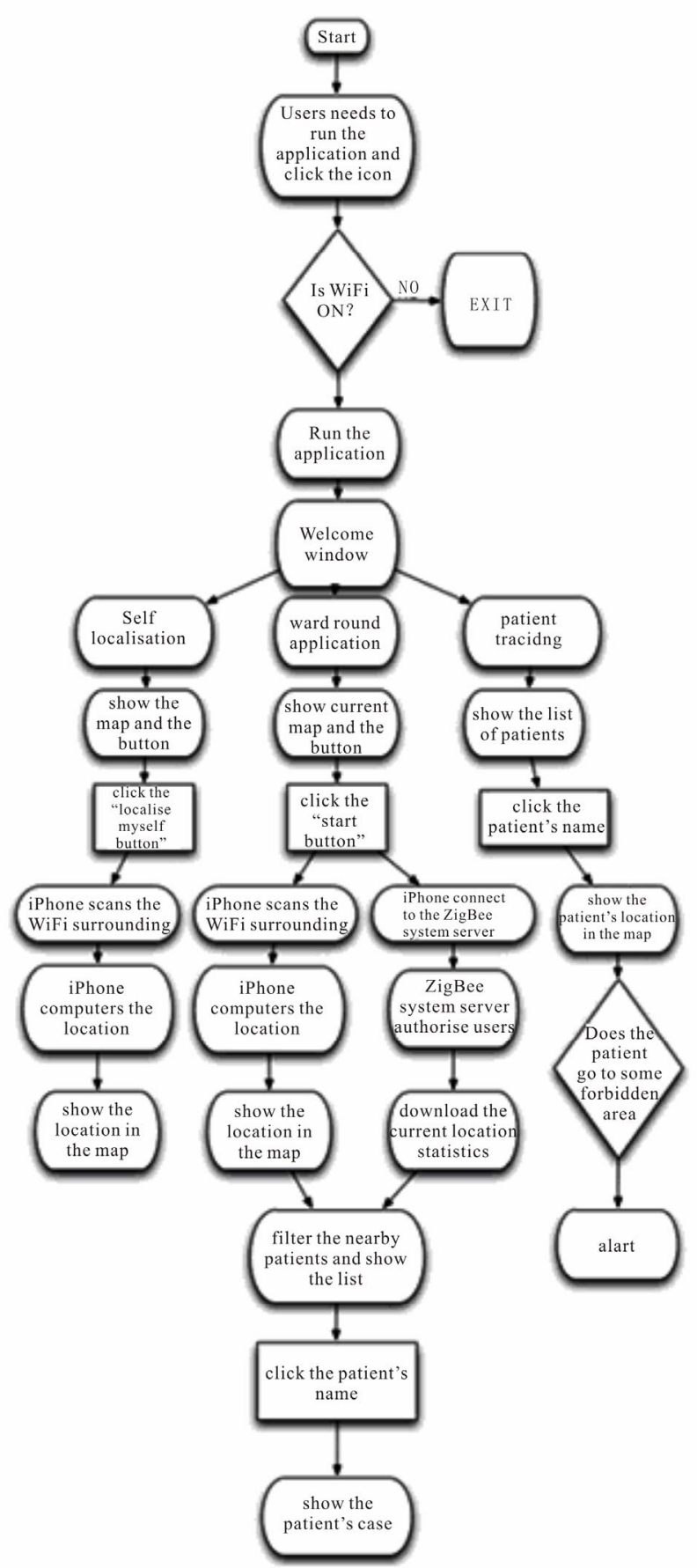

Figure 1. Overall architecture.

has stopped disclosing its public APIs for obtaining the network's information. One possible way to get current RSSI value is put all APs into monitor mode. It will send signal with RSSI strength according our programming. But the obvious drawback of this method is that all APs cannot connect to the Internet, which means that these APs just provide localization function. In our system, we have found a way to solve this problem. Instead of using monitor mode APs, we install the application in root 
folder of iPhone operation system, which makes it can use iPhone private API. By using this way, it simplifies the APs configuration. However the disadvantage is that this software must be setup by trained people.

Before we use the WiFi-localization, we should execute calibration phase. In calibration phase, iPhone will detect surrounding WiFi signal to record them as offline data. In online phase, according different algorithms, iPhone will execute different methods to compute the current location by comparison of recorded statistics.

\subsection{ZigBee Localization System}

Figure 2 shows the structure of ZigBee localization system. The main components of this system are: 1) Asset tags; 2) Sensors of fixed location; 3) Base station which will receive data from sensors; 4) Database.

It contains two parts: hardware and software.

In this project, CC2530 evaluation modules are employed as tag and readers and CC2531 USB dongle is used as base station to transmit information captured from readers to PC (Figure 3). Furthermore, serial port connection plays a key role in hardware part. It takes charges of transmit received statistics to database.

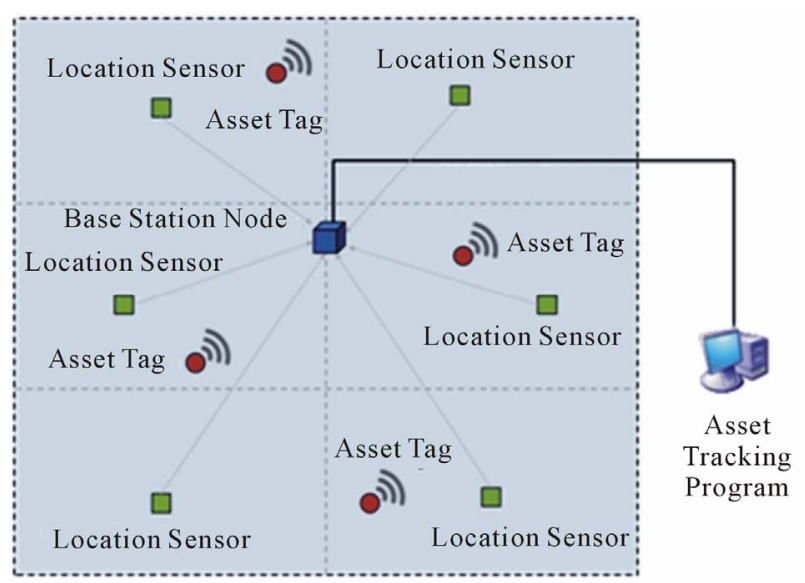

Figure 2. ZigBee system architecture.

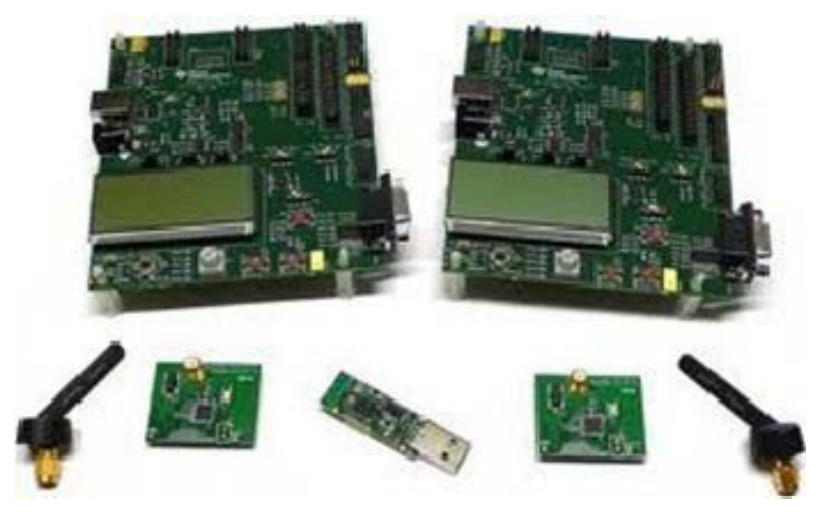

Figure 3. CC2530.
In software part, $\mathrm{C \#}$ and Microsoft SQL are used in programming. The localization algorithm of this system is described as follow. It is a classified as a range-free localization algorithm. The sensors with fixed location will detect whether there is a tag in their detection zone.When multiple location sensors can scan a same tag, the maximum RSSI value received by the location sensor is used to determine the detection zone.

Calibration phase, as mentioned previously, makes use of comparison between recorded map and current value. Because of unstable asset tag measured result, a probability method is adopted.

\section{Algorithms and Experiment}

\subsection{WiFi Localization System [6,7]}

Since this paper focuses on WiFi localization application on iPhone, this section will compare and analyze the performance of different localization algorithm on iPhone. We will also briefly introduce the ZigBee localization system in the second part of this section. In this project, the WiFi system uses three different algorithms: nearest neighbor, K-nearest neighbor, probability.

We did experiment in a lab. The floor plan was plotted in Figure 4. As we can see, there are three main APs in this lab. But we still could scan more than five WiFi signals, which also could be used to localization. For WiFi

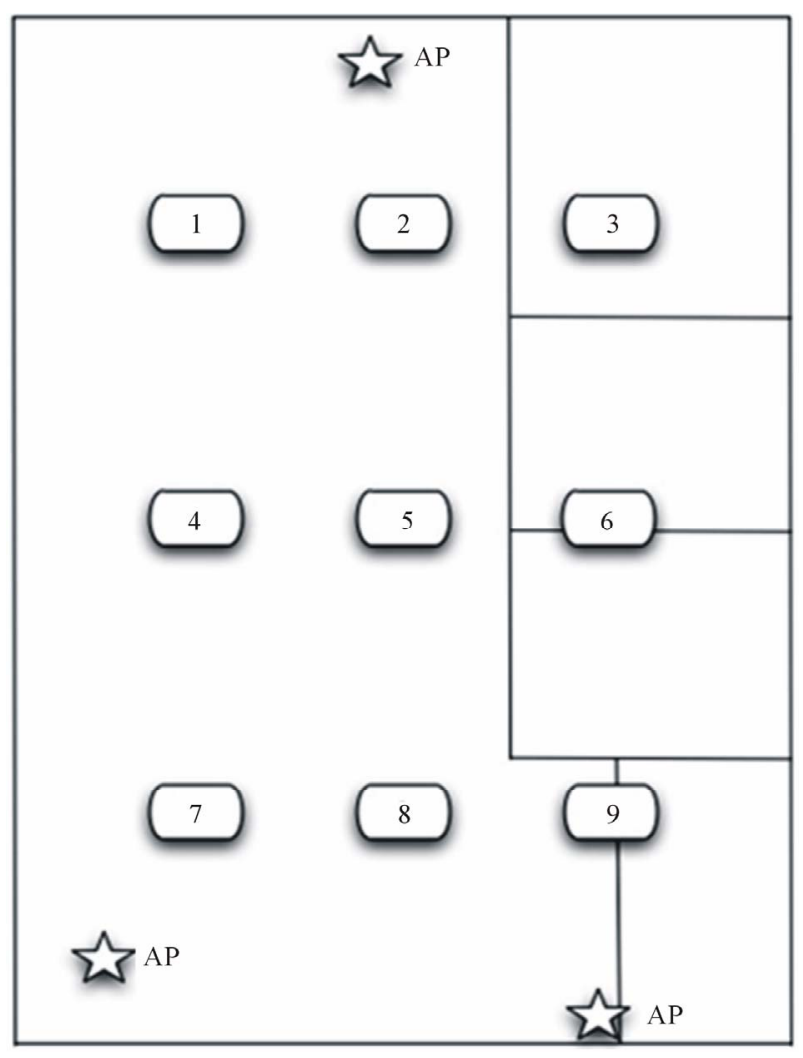

Figure 4. Floor plan. 
localization phase, we don't have to know the WiFi password. As long as we can scan the signal, we can use it. According our experience, the more APs the more accurate. Hence, in WiFi phase, if we want to continue to improve the accuracy, we just simply increase the number of main APs instead of changing the algorithm.

Offline phase based on IEEE 11 channel model: In the calibration phase, a radio map of the environment is created. A radio map is a database of locations throughout the environment with RSSI value, MAC address, channel number and SSID. The process of producing a radio map based on actual measurements is not only labor-intensive and costly but also very sensitive to changes in the environment and possible effects of interference in the building. Using IEEE 802.11 channel model can be a simple alternative way to create a radio map.

To provide a better presentation of our environment, we have used IEEE 802.11 channel model in model C. The received power in IEEE 802.11 channel model is represented by (1).

$$
R S S(d)=P t-L(d)+f(d)
$$

where, $P t$ is the transmitting power, $d$ is the distance between the sender and receiver. The $f(a)$ stands for all kinds of fading. In this situation, the main fading is the shadow fading process which given by (2) and (3) respectively,

$$
\begin{gathered}
L(d)=L_{0}+10 a_{1} \log 10(d) \quad d \leq d_{B P} \\
L(d)=L_{0}+10 a_{1} \log _{10}(d)+10 a_{2} \log _{10}\left(\frac{d}{d_{B P}}\right) \quad d \leq d_{B P}(2) \\
f(d)=\rho \cdot f(d-1)+\sqrt{\left(1-\rho^{2}\right)} \cdot N(0, \sigma)
\end{gathered}
$$

where, $L_{0}$ : the path loss at reference distance. $d_{B P}$ : the breakpoint distance in meters $(1 \mathrm{~m}) . a_{1}$ : is the powerdistance gradient before the breakpoint. $a_{2}$ : is the powerdistance gradient after the breakpoint. $\sigma$ : standard deviation. $\rho$ : a correlation coefficient.

Nearest neighbor algorithm: This is the simplest algorithm, which will release utilization of iPhone CPU. For experiment, we draw a floor plan in iPhone. Meanwhile, to execute the offline phase, we design nine points in the floor plan to record the WiFi signal strength (RSSI value), MAC address of APs, sequence number of channel and SSID of APs for calibration and story it into iPhone default database, which will automatically exist in the software when users download their software and never missing.

When the software runs the nearest neighbor algorithm, it follows below equation:

$$
d=\sqrt{\left(s s_{1}-S S_{1}\right)^{2}+\left(s s_{2}-S S_{2}\right)^{2}+\cdots+\left(s s_{n}-S S_{n}\right)^{2}}
$$

where $s s_{n}$ is current RSSI value of nth AP. $S S_{n}$ is calibration recorded RSSI value of nth AP.

After all of distance is computed, it will choose the highest value of Euclidian distance as its position and map it.

K-nearest neighbors algorithm: To improve the performance and accuracy of $\mathrm{NN}$ algorithm, the common way is using KNN. Instead of using all of RSSI value, KNN algorithm chooses K samples of Euclidian distance which are defined by follow equation:

$$
E_{i}=\sqrt{\sum_{j=1}^{N}\left(s S_{i}-S S_{i}\right)}
$$

After sorting the calculated $E_{i}, \mathrm{~K}$ data samples with smaller Euclidian distance are picked up as $\mathrm{K}$ nearest neighbors. $\mathrm{K}$ is usually an integer number between 1 and $N$ samples. To map the position, the position with smallest Euclidian distance will be selected as the final position.

Probability algorithm: Unlike NN and KNN, probability depends on the probability of RSSI value [8]. Because we try to complete all of calculation in iPhone, it is inconvenient to implement the probability algorithm we used in ZigBee localization system, which will increase the load of CPU of iPhone and affect the real time localization ability. In this WiFi localization system, we try to use a simple probability algorithm.

When we do the training method, we can record multiple times RSSI value for every WiFi signal. Then, when users try to use the application, it will scan current WiFi signal strength to check the probability that there is a same value in recorded data. The position with the highest sum of probability will be mapped in the screen.

Experiment measurement and result: We have done 20 times test at 9 positions for every phase. Then we calculate the error of every time and time-consumption. Then we plot the CDF (Cumulative Distribution Function).

Error:

$$
\operatorname{error}(\mathrm{cm})=\sqrt{\left(x_{\text {actual }}-x_{\text {test }}\right)^{2}+\left(y_{\text {actual }}-y_{\text {test }}\right)^{2}}
$$

According above Table $\mathbf{1}$ and Figures $\mathbf{5}$ and $\mathbf{6}$, we can conclude:

1) $\mathrm{NN}$ is the simplest way to localize, but it has the worst performance.

2) KNN and probability phase have similar performance.

3) This localization system owns better accuracy in the area which is far from wall especially for KNN. Because in position 4 and 7 of $\mathrm{KNN}$, the accuracy of 20 times test can reach perfection.

4) Compared with $\mathrm{NN}$ and $\mathrm{KNN}$, the probability phase is the highest complex, which means that it needs much more time consumption.

5) Though KNN just calculate $\mathrm{K}$ nearest neighbor, but it still costs more time than NN phase because of the 
Table 1. Error distance.

\begin{tabular}{cccc}
\hline & $\mathrm{NN}$ & $\mathrm{KNN}$ & Probability \\
\hline 1 & 568.501 & 244.566 & 234.078 \\
2 & 438.353 & 443.194 & 263.309 \\
3 & 510 & 502.209 & 553.559 \\
4 & 403.5 & 0 & 39 \\
5 & 80.077 & 256.040 & 267.622 \\
6 & 168 & 474.141 & 341.941 \\
7 & 622.383 & 0 & 187.5 \\
8 & 418.373 & 143.012 & 305.273 \\
9 & 168 & 382.5 & 157.5 \\
Average & & & 345.078 \\
error & 375.242 & 224.566 & \\
distance & & & $3826 \mathrm{~ms}$ \\
Average & & $1871 \mathrm{~ms}$ & \\
time & $1805 \mathrm{~ms}$ & & \\
consumption & & & \\
\hline
\end{tabular}

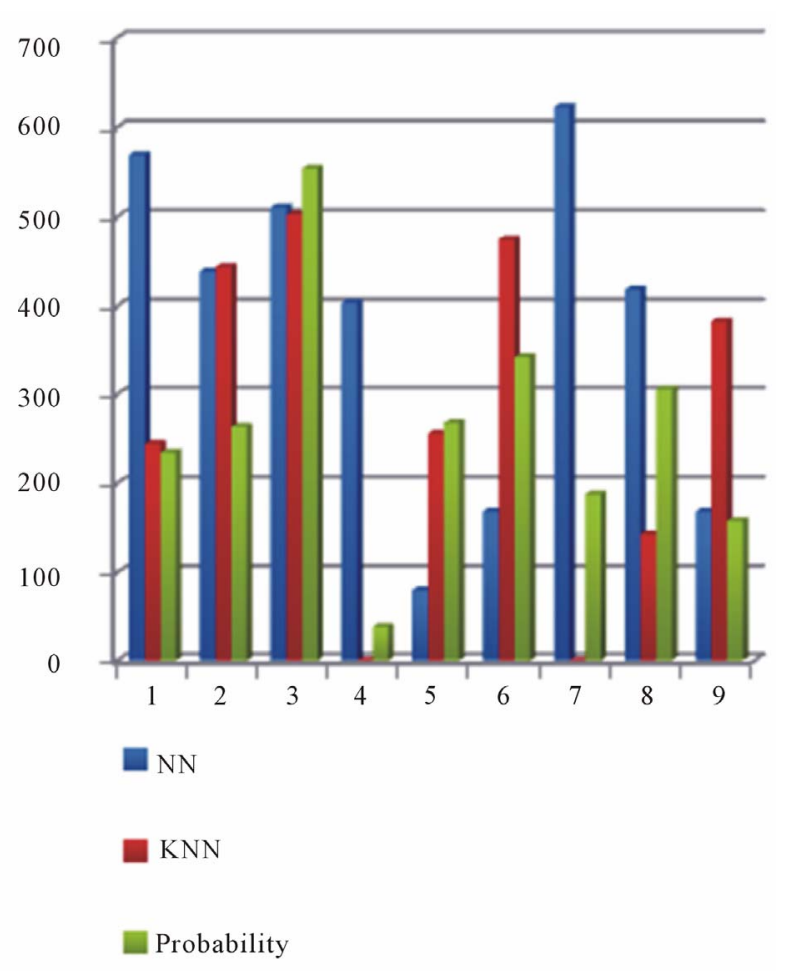

Figure 5. Position error distributions.

sorting process.

6) According the Figure 6, probability phase has better performance than others. More than $50 \%$ localization points have no error. However, NN phase has the worst error statistics. More than $50 \%$ localization points have above $400 \mathrm{~cm}$ error distance.

7) For the health care system, since we are focusing on not only the accuracy and but real-time feature, we select KNN as our application phase.

Although the test result was good, we still can find that the position of current location may jump to other places

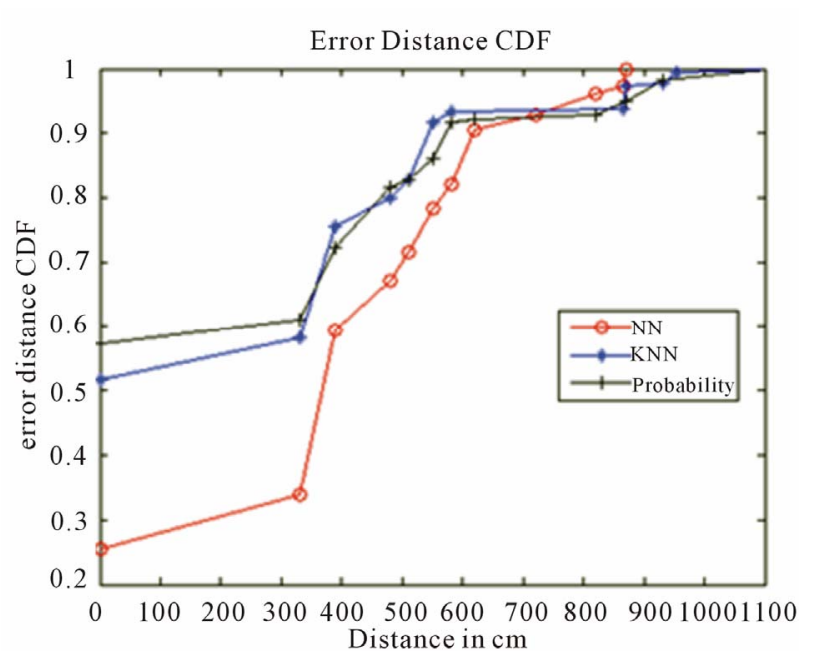

Figure 6. Error distance CDF.

instantaneously, which has bad performance. Thus, to improve the accuracy, we added a filter to the last result.

As the above introduction, the RSSI value may be affected by change of circumstance. For instance, if there are many people around the receiver, the RSSI value can change dramatically. According our test, the received RSSI can have $10 \mathrm{~dB}$ differences at a same position. Hence, to solve this problem, we store the last several times results as a reference statistics. If the final result is different from the previous data, we should make a decision whether the position should be changed. The logic of the filter is following the diagram Figure 7.

Furthermore, to reduce the chance that the display of the position will jump a large-scale distance, we introduced a small-scale jump phase. Instead of jumping to the destination point directly, in our system, the position will just move to the inferred point with smaller distance.Because, in our system, the calibration points in the radio map have 397.5 centimeters distance. Although walking speed is various depending on the height, weight, age, terrain, surface, load and so on. The average speed is 5 kilometers per hour, or about 1.3 meters per second. So, imagining a person is walking in our experimental circumstance, the average variation is almost one third of distance of changing.

$$
\begin{aligned}
\text { Inferred Position } & =\text { Original Position } \\
& +\frac{\text { Distance Difference }}{3}
\end{aligned}
$$

where, Distance Difference is the distance between last position and current measured position.

Thus, we can track the moving of inferred position. From the above analysis, we know that the probability phase has better performance for the reason that it uses more record statistics. Thus, to improve the NN, KNN performance, we change the calibration phase. Instead of recording the data directly, we adopt a method that it 


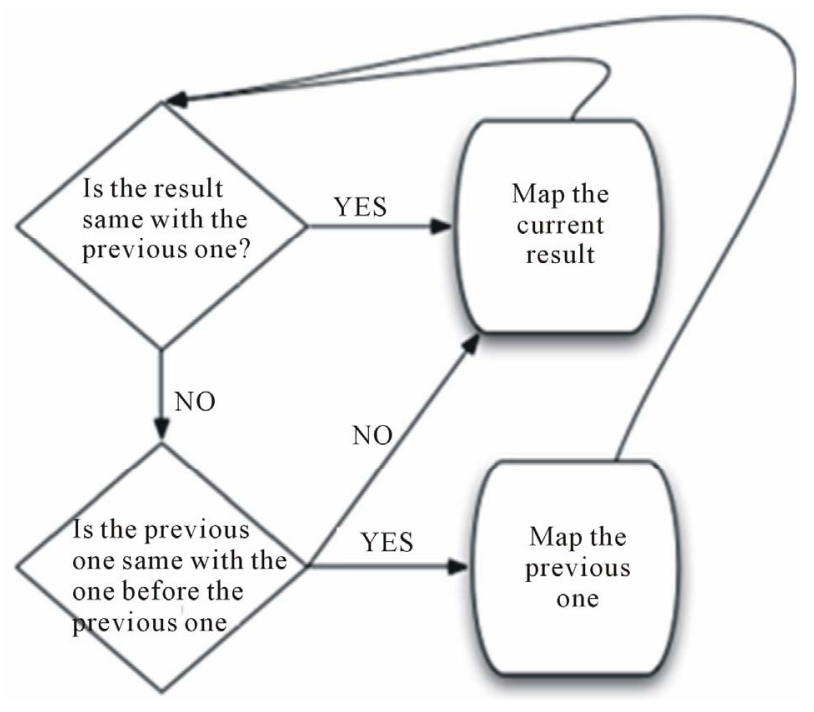

Figure 7. Logic of filter.

scans the WiFi signal $\mathrm{n}$ times and calculate the average RSSI value. In this way, it will avoid the break changing of RSSI value. Meanwhile, to reduce the effect of the direction of devices, we can scan the multi-direction in calibrationphase. When we do the calibration, the user should go round in the position to collect all the statistics of multi-direction.

Because we have found that the probability phase has obvious delay. Hence, we just implement above filters and phases in NN and $\mathrm{KNN}$.

Then, we did the calibration phase and tested the error distance again just like the previous one. The final experimental resultis as follow:

From the final experimental result (Table 2, Figures 8 and 9), we can see that the average error distance of KNN can be reduced to $123.7 \mathrm{~cm}$ by mentioned filters and phases. The time consumption will increase a little from $1871 \mathrm{~cm}$ to $1925 \mathrm{~ms}$. In addition, because the position will move small distance every time when the current position is different from the previous one, the number of small error distance increases. We can see this phenomenon from Figure 9. However, the performance of $\mathrm{NN}$ and $\mathrm{KNN}$ with filters gets improvement. From Figure 9, the performance of NN with filters is even better than the performance of probability without filters. It also shows that more than 90 percent of KNN inferred positions with filters have error distance less than 3 meters.

The reason why filters can improve the accuracy is that it filters unstable statistics in the radio map. Hence less unexpected jump happens. In addition, the step filter measures the position according human move, which can reduce the large-scale jump.

There are still two factors we can explore their relationships with performance, which are the $\mathrm{K}$ value of $\mathrm{KNN}$ and the number of sampling points.
Table 2. Error distance with filter.

\begin{tabular}{ccc}
\hline \multirow{2}{*}{ Position Index } & \multicolumn{2}{c}{ Error distance } \\
\cline { 2 - 3 } & NN & KNN \\
\hline 1 & 329 & 77 \\
2 & 214 & 129 \\
3 & 251 & 62 \\
4 & 480 & 56 \\
5 & 270 & 268 \\
6 & 77 & 23 \\
7 & 416 & 76 \\
8 & 91 & 293 \\
9 & 49 & 128 \\
Average error & 242 & 124 \\
distance & & $1925 \mathrm{~ms}$ \\
Average time & $1845 \mathrm{~ms}$ & \\
consumption & &
\end{tabular}

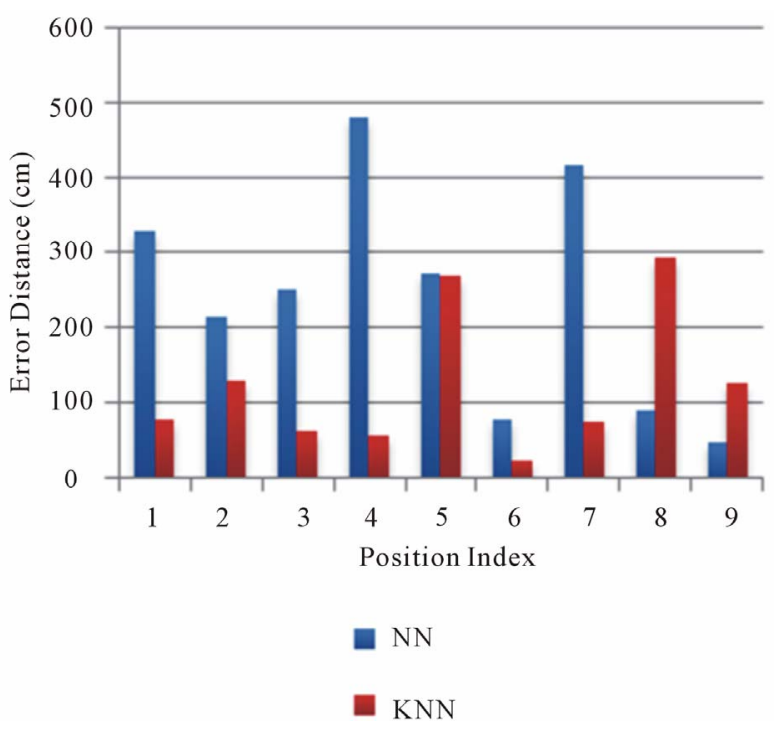

Figure 8. Position index.

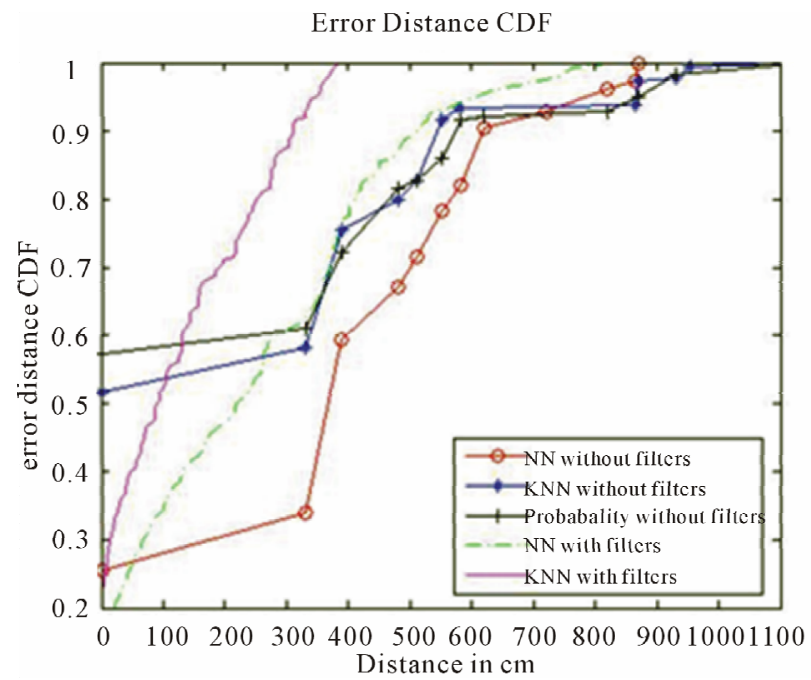

Figure 9. Error distance CDF. 
We tested 9 sampling points' case, and got the statistics: From Table 3 and Figure 10, we can know that the $\mathrm{K}=4$ is the best algorithm for localization in 9 sampling points' case. Furthermore we can find the average time consumption will increase with the value of $\mathrm{K}$.

Next, for 16 sampling points' measurement, we got the experimental statistics as follow: From Table 4 and Figure 11, we can easily find that $\mathrm{K}=4$ is more accurate than others. What's more, the time consumption still follows the law we got in 9 sampling points. And we also can find that the 16 sampling points will cost more time than 9 sampling locations case.

Finally, we tested the 25 sampling points, which is much more complex than others (Table 5). \#We plotted the average error distance at every location:

From Figure 12 and Table 5, we find that the result is different from the earlier two cases. $\mathrm{K}=5$ has the best result. The reason of this is that the 5th signal is stationary at the time of the experiment. Furthermore, 25 sam pling points case will be sensitive to any small variation, so $\mathrm{K}=4$ cannot be as good as 16 sampling points case and 9 sampling points.

From all these 9 tests, we can get the following conclusions:

$\mathrm{KNN}$ with $\mathrm{K}=4$ has better result than others generally. 16 sampling points is the best situation.

9 sampling points' case and 25 sampling points' case have similar performance in average error distance,

Table 3. 9 sampling test statistics.

\begin{tabular}{cccc}
\hline & \multicolumn{3}{c}{ Error distance } \\
\cline { 2 - 4 } & $\mathrm{K}=3$ & $\mathrm{~K}=4$ & $\mathrm{~K}=5$ \\
\hline $\begin{array}{c}\text { The Worst Error } \\
\text { Distance } \\
\text { Average Error } \\
\text { Distance }\end{array}$ & 379 & 293 & 587 \\
$\begin{array}{c}\text { Average time } \\
\text { consumption }\end{array}$ & 170 & 124 & 186 \\
\hline
\end{tabular}

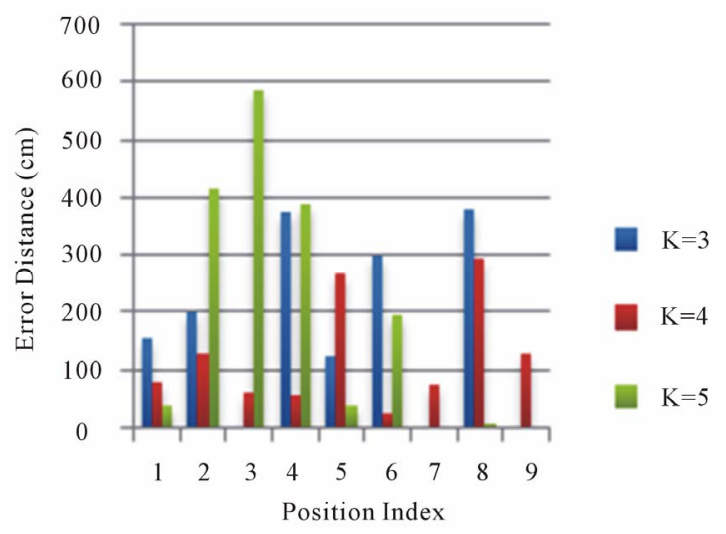

Figure 10. Average error distance at every location for 9 sampling points.
Table 4. 16 sampling points test statistics for 9 sampling points.

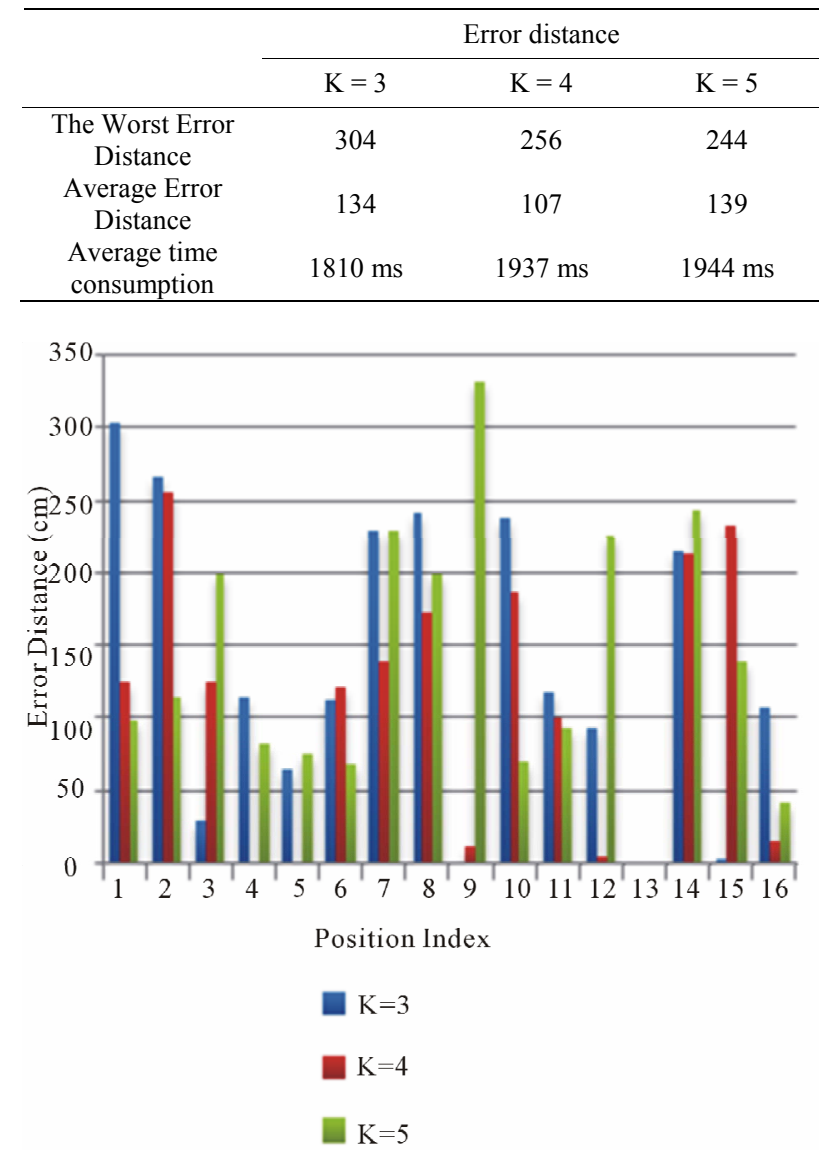

Figure 11. Average error distance at every location for 16 sampling points.

Table 5. 25 sampling test statistics.

\begin{tabular}{cccc}
\hline & \multicolumn{3}{c}{ Error distance } \\
\cline { 2 - 4 } & $\mathrm{K}=3$ & $\mathrm{~K}=4$ & $\mathrm{~K}=5$ \\
\hline $\begin{array}{c}\text { The Worst Error } \\
\text { Distance }\end{array}$ & 482 & 475 & 340 \\
$\begin{array}{c}\text { Average Error } \\
\text { Distance }\end{array}$ & 183 & 179 & 158 \\
$\begin{array}{c}\text { Average time } \\
\text { consumption }\end{array}$ & $1891 \mathrm{~ms}$ & $1971 \mathrm{~ms}$ & $2663 \mathrm{~ms}$ \\
\hline
\end{tabular}

which are worse than 16 sampling points. Although 25 sampling points is more complex, but the statistical similarity is too high to get accurate result.

$\mathrm{K}=3$ has the least time-consumption, because after selecting $\mathrm{k}$ minimum error distance, it only uses the 3 minimum error distance to localize.

For time consumption, 9 sampling points $<16$ sampling points $<25$ sampling points. The reason for this phenomenon is that, for 9 sampling points' case, it only calculates the distance between current location and 9 recording calibration locations. But for 25 sampling points, it has to compare with 25 recording calibration 


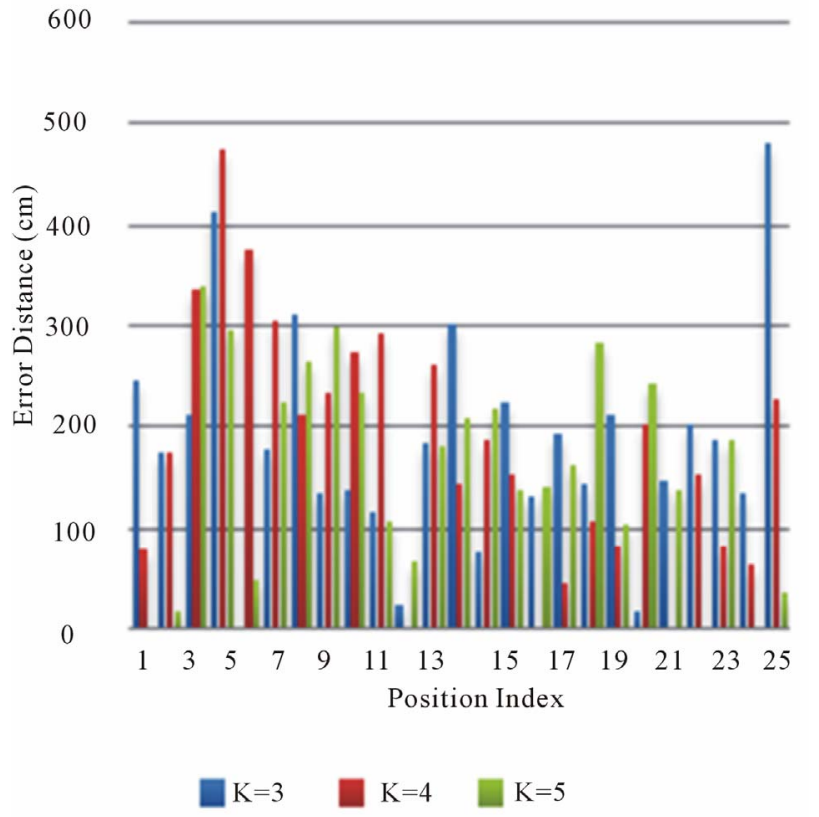

Figure 12. Average error distance at every location for 25 sampling points.

\section{locations (Figure 13).}

We can also find that the bottom points have better accurate than others. The bottom points mean that the measured points near the bottom line of the floor plan. The reason of this result is that around the bottom line there are two APs, which can localize the location accurately. At the top of the floor plan there is only one AP. The system hardly finds the accurate location. To improve the accuracy of this experimental circumstance, we should add one AP at the top line of the floor plan.

16 sampling points $\mathrm{KNN}$ algorithm with $\mathrm{K}=4$ can achieve $107 \mathrm{~cm}$ error distance accuracy.

To research the relationship between the $\mathrm{K}$ value of $\mathrm{KNN}$ and its localization performance, we tested three situations with $\mathrm{K}=3, \mathrm{~K}=4, \mathrm{~K}=5$, and plotted their performance. According to previous literature, the $\mathrm{K}$ value depends on the algorithm, the measurement method and circumstance. We plotted the CDF figures of three cases (Figures 14-16).

From the 9 sampling points, we can know that KNN with $\mathrm{K}=4$ is the best algorithm.

From the 16 sampling points, we can know that KNN with $\mathrm{K}=4$ is the best algorithm.

From the 25 sampling points, we can know that KNN with $\mathrm{K}=4$ has outstanding performance.

According to Figure 14-16, we can get the conclusion:

Totally, $\mathrm{K}=4$ has better performance than others. Because, in our experimental circumstance, there are four major routers, they can provide the strong evidence for localization. For RSSI value, the closer distance between

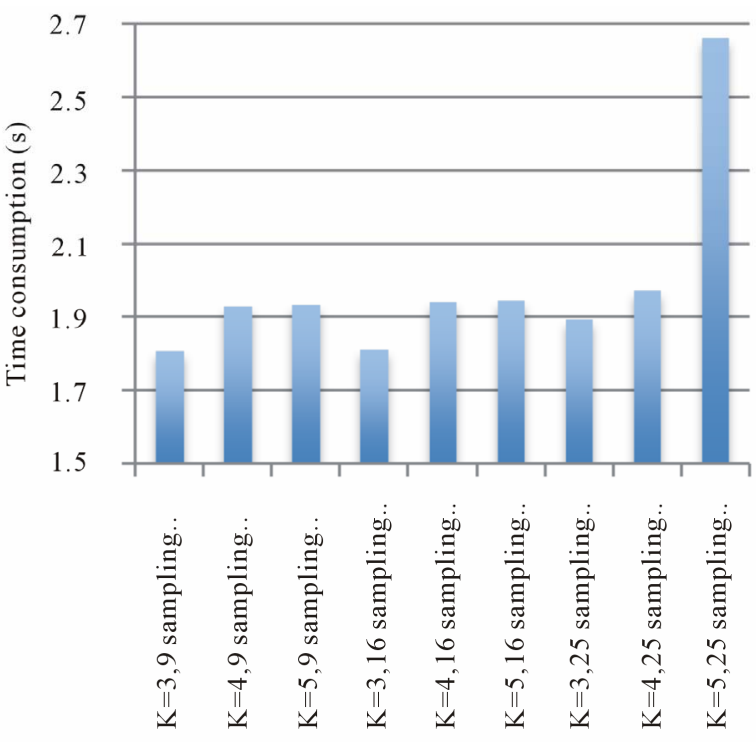

Figure 13. Time consumption of each situation.

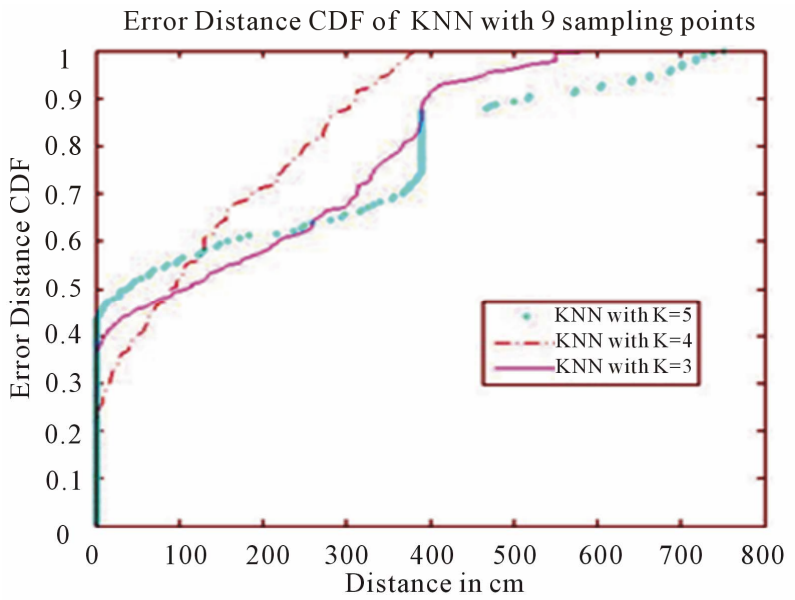

Figure 14. 9 sampling points with different $K$ value.

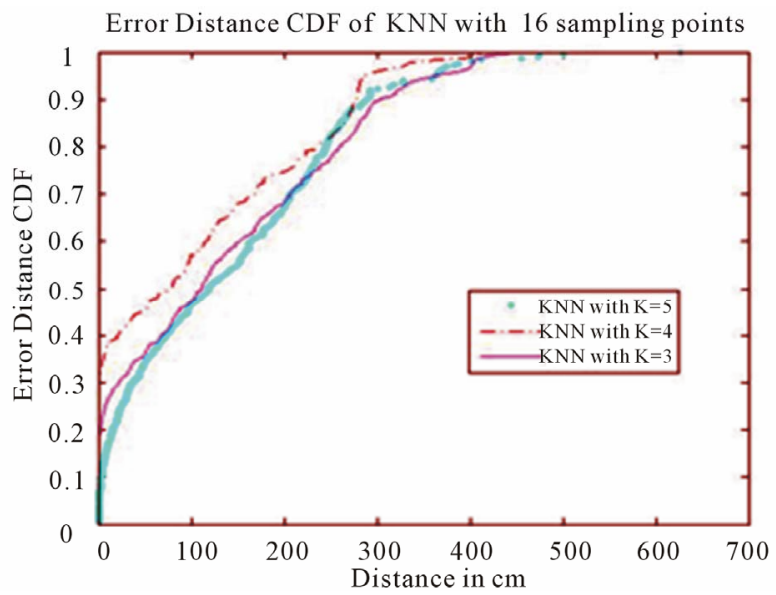

Figure 15. 16 sampling points with different $K$ value. 
Error Distance CDF of KNN with 25 sampling points

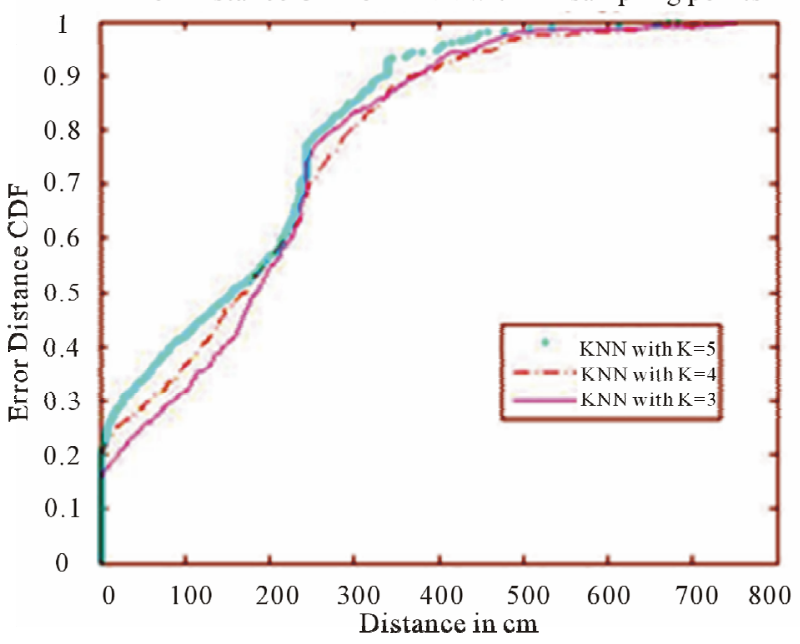

Figure 16. 25 sampling points with different $K$ values.

APs and receivers, the better stable performance we will get.

For $\mathrm{K}=5$ cases, in 25 sampling points, it has better accuracy than 9 sampling points' case and 16 sampling points' case. Because, in 25 sampling points, neighbor distance is smaller than others, it protects against smallscale variation. For the 5th strongest signal, it is not the major signal. Hence, it will experience lots of reflection, diffraction and multipath. It also passes several walls and floors. We can use Ericsson multiple breakpoint model [9] to estimate the remote APs signal.

In 9 sampling points, the error distance for different $\mathrm{K}$ values is fluctuating. The reason of this phenomenon is that signal surrounding of sampling points in 9 sampling points' case are quite different. And the distance between points is large. Hence long-distance error can be happened.

To research the relationship between the sampling density and its localization accuracy, we also change the sampling density. Instead of 9 sampling points, we use 16 sampling points and 25 sampling points (Figure 1719) Then we compare their performance and get the conclusion.

We measured minimum distance between two neighbors in there cases:

We plotted the above three situations in Figure 20. Then we compared their performance.

From Figure 20 and Table 6, we can know that, for short-term distance error, 9 sampling points' case has better performance than others. For long-term distance error, 16 sampling points' case is the best one rather than 25 sampling points' case.

We can also find the result of Figure $\mathbf{2 1}$ is similar with the previous one. The difference is that in short-term distance error 16 sampling points' case has similar performance with 9 sampling points' case.

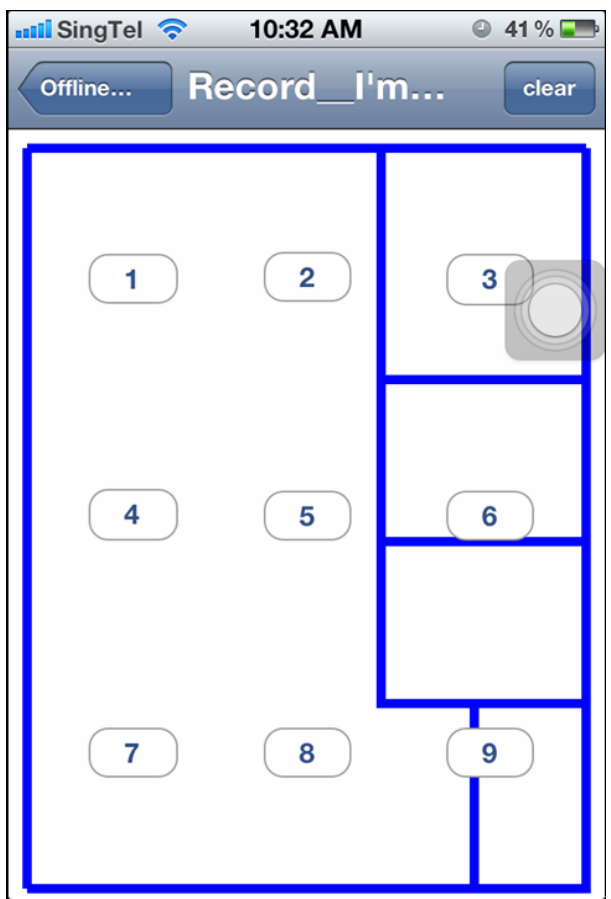

Figure 17. 9 sampling points.

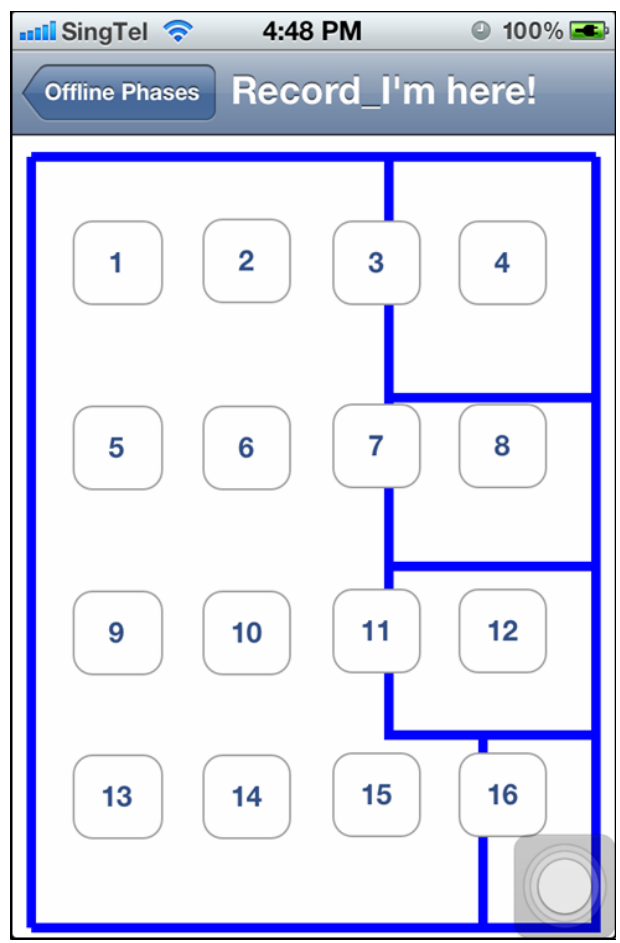

Figure 18. 16 sampling locations.

For $\mathrm{K}=5$ case, we can also find the conclusion. But the result is clearer than others. In short-term distance error almost half of result in 9 sampling points is zero error. But in long-term case, 16 sampling points is brilliant.

From Figures 20-22, we can get the conclusion: 


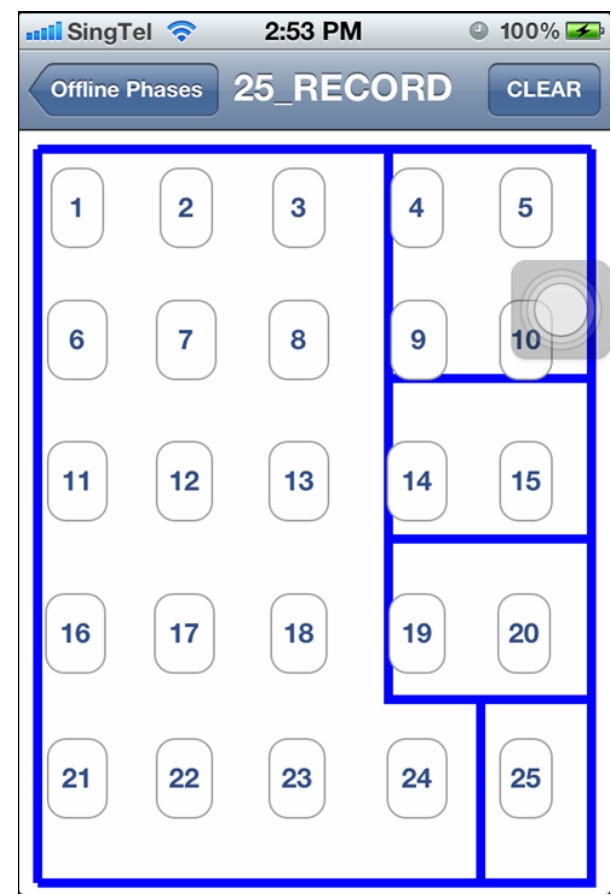

Figure 19. 32 sampling locations.

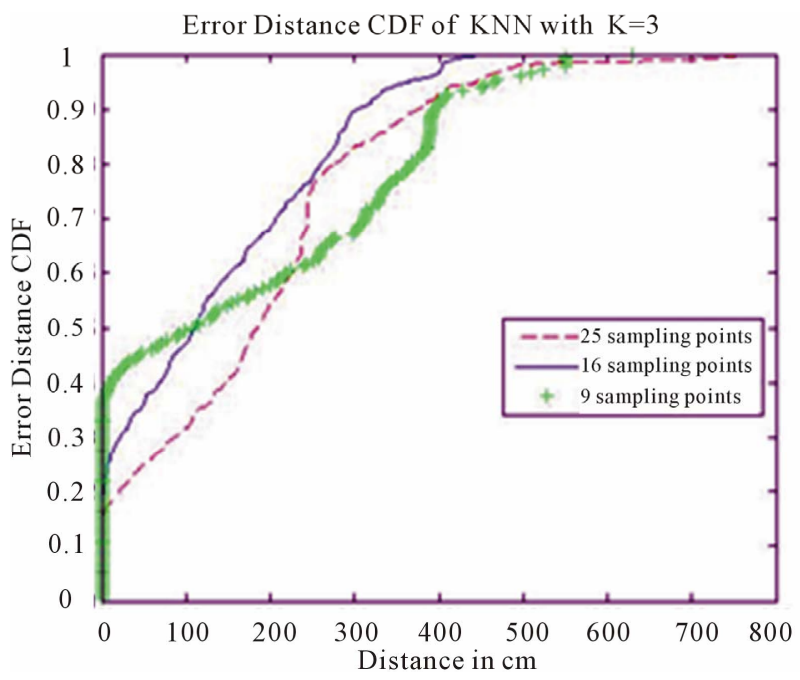

Figure 20. Different sampling points with $K=3$.

Table 6. Minimum neighbors distance.

\begin{tabular}{lccc}
\hline & $\begin{array}{c}9 \text { sampling } \\
\text { points }\end{array}$ & $\begin{array}{c}16 \text { sampling } \\
\text { points }\end{array}$ & $\begin{array}{c}25 \text { sampling } \\
\text { points }\end{array}$ \\
\hline $\begin{array}{c}\text { Minimum distance } \\
\text { between two } \\
\text { neighbors }(\mathrm{cm})\end{array}$ & 330 & 312 & 260 \\
\hline
\end{tabular}

9 sampling points has better accuracy or more zero distance in short-term distance error. As we know, 9 sampling points case has far neighbors. It has slim chance to skip to their neighbor, because their neighbors have quite different WiFi circumstance. From minimum neighbors' distance table, we have gotten the minimum distance between two neighbors. For 9 sampling points' case, the minimum distance is $330 \mathrm{~cm}$, which is larger than others.

However, 16 sampling points' case and 25 sampling points' case have outstanding accuracy in long-term. For small probability error, which is long-term distance error, 16 sampling points' case and 25 sampling points case have common feature that they can protect against the happen of long-term distance error. The reason of this phenomenon is that, for these two cases, they have closer neighbor than 9 sampling points' case. Even if the measured signal strength is quite different from the calibration strength, it can also find near neighbor to localize.

16 sampling points' case is more accurate than 25 sampling points. For 25 sampling points, the minimum neighbors distance is too little to localize correctly. The relevant coherency of signal strength between neighbors

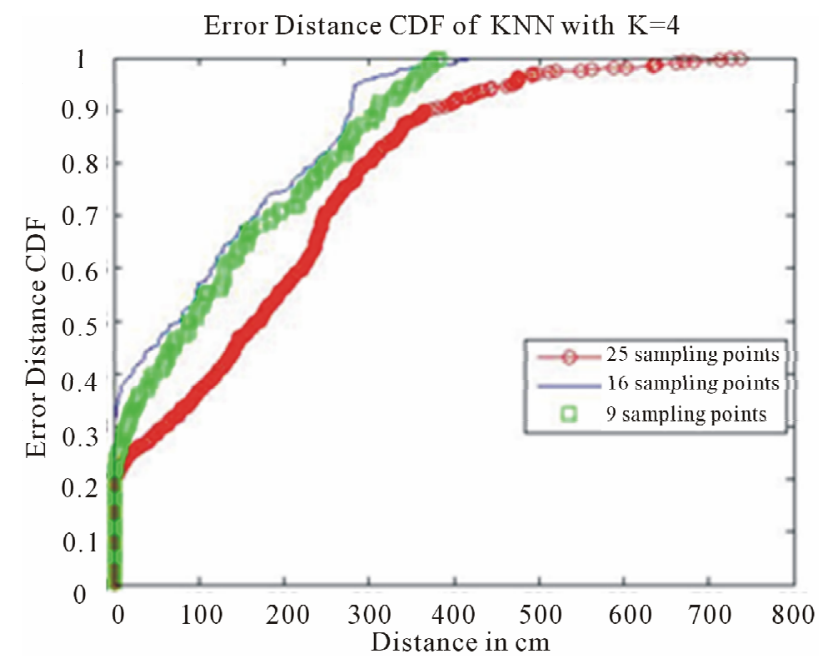

Figure 21. Different sampling points with $K=4$.

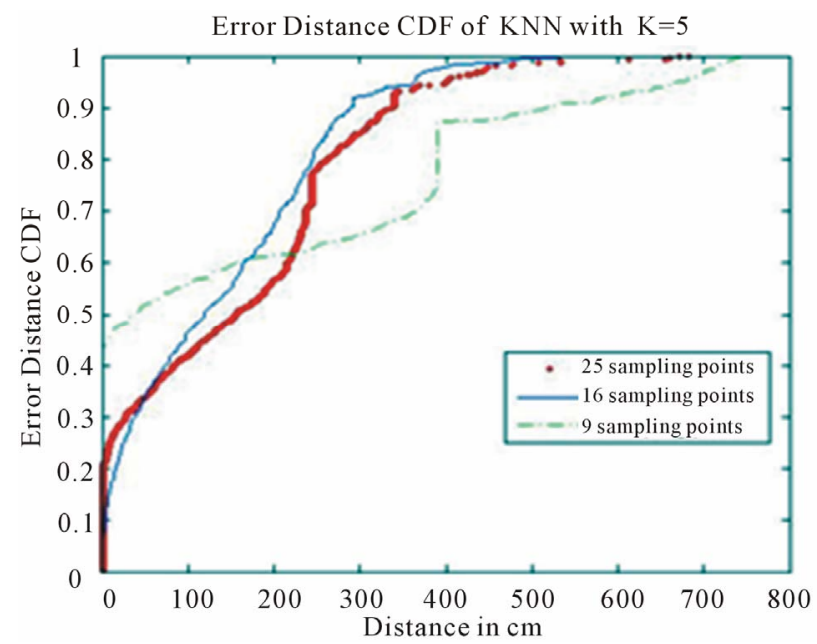

Figure 22. Different sampling points with $K=5$. 
affects its performance. We have already known that, even in same situation, the received strength of same signal source will be different. The range of that is \pm 10 $\mathrm{dB}$. So for 25 sampling points, it has large probability to skip to its neighbor locations.

To find the optimization, we plotted overall CDF figure (Figure 23).

We can find the 16 sampling points $\mathrm{KNN}$ with $\mathrm{K}=4$ is the best algorithm. To find the optical number of calibration points, we can find the average area for each point.

Our experimental lab is $1560 \mathrm{~cm} \times 1620 \mathrm{~cm}=$ 2,527,200 $\mathrm{cm}^{2}$. Hence, for each point of 16 sampling points case, we separate the room into 16 areas. Each point covers $2,527,200 \div 16=157,950 \mathrm{~cm}^{2}=15.795 \mathrm{~m}^{2}$. So we can get the conclusion that, to install localization system like our experimental circumstance, each separated area should cover about $16 \mathrm{~m}^{2}$ and do calibration phase.

For value of $\mathrm{K}$, we can get conclusion that the number of $\mathrm{K}$ value should be equal to the major APs you have.

This system has two subsystems: doctor's subsystem and patient's subsystem.

For patient's subsystem, because this system is used as self-localization and self-navigation, as users, they can clearly tell the small distance error and subjectively tell which is the correct location. We just care about longterm distance error. From Figure 23, we can know 16 sampling points $\mathrm{KNN}$ algorithm with $\mathrm{K}=4$ is the best one. We will adopt this method in our healthcare application in chapter $\mathrm{V}$.

For doctor's subsystem, because it is used as ward round case filter, it should be very accurate in small-term distance and of high-resolution ratio to tell the difference form patient to patient. Thus we selected 25 sampling points with $K=4$ as the algorithm.

Since there are lots of other future functions of this application, we can select different algorithm according to the requirement of the application.

\subsection{ZigBee Localization System [10]}

In the ZigBee localization system, we used Improved Bayesian probability radio map algorithm. Because we did this in the server, we needn't care more about the complexity of the algorithm. Thus, improving the accuracy is the target.

We assume that the value received by every location sensor belongs Gaussian Distribution. The equation for mean and deviation is shown below:

$$
\begin{gathered}
\mu=\frac{\sum_{i=1}^{n} x_{i}}{n} \\
\sigma=\sqrt{\frac{\sum_{i=1}^{n}\left(x_{i}-\mu\right)^{2}}{n}}
\end{gathered}
$$

$$
f(x)=\frac{1}{\sigma \sqrt{2 \pi}} \mathrm{e} \frac{(x-\mu)^{2}}{2 \sigma^{2}}
$$

where $x_{\mathrm{i}}$ is an RSSI value; $\mu$ is the mean value of sample RSSI value $\sigma$ is the standard deviation of sample RSSI value.

According the Gaussian Distribution, when the base station receives a RSSI packet, it can compute the probability of current value in each location sensor. Finally the position with highest probability will be assigned to the final position.

Improved Bayesian Probability Radio Map Algorithm [10]:

The probabilistic approach for radio map algorithm can be realized by using the concept of, conditional probability, Bayes theorem (Figure 24). The formula used for computing the likelihood for each position of the asset tag is shown below:

$$
P\left(A_{i} \mid B\right)=\frac{P\left(B \mid A_{i}\right) \times P\left(A_{i}\right)}{\sum_{j}^{n}\left[P\left(B \mid A_{j}\right) \times P\left(A_{j}\right)\right]}
$$

where: $\mathrm{N}$ is the number of location points of the radio map; $P\left(A_{i}\right)$ is the probability that the object is at the particular point $\mathrm{I} ; P\left(B \mid A_{j}\right)$ is the probability that the particular RSSI value $B$ is received at a particular point I; $P\left(A_{i} \mid B\right)$ is the probability that the object is in a particular point I given the received RSSI value $B$.

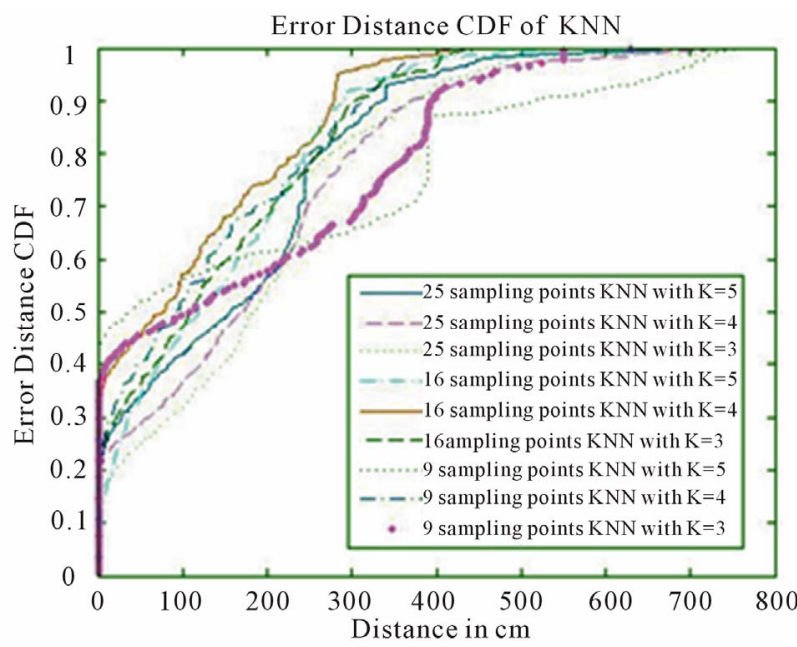

Figure 23. Overall performance.

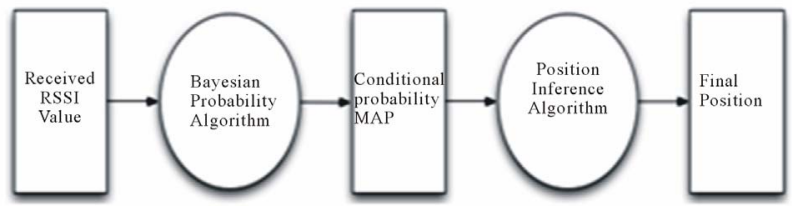

Figure 24. Block diagram of improved bayesian probability radio map algorithm. 
When the reader received the RSSI value, it would first convert the values into a probability value for each calibration point using "probabilistic map". A "conditional probability map" could then be calculated by using the probability values from the previous step. A conditional probability map contained probability that the asset tag would be for each calibrated point shown below. The position of the highest probability would be deemed as the position of the object.

Position Inference Algorithm: The algorithm would take into consideration a number of calibration points that locate near the test point, instead of just the nearest calibration point, during computation shown below. It was based on the assumption that points, which were located close together, had relatively similar RSSI signature values.

The final "inferred" position was then computed in a function that involved the probability of each points and distances between the considered points.

To improve the accuracy, we combined the two algorithms. The result of the nine test points has shown in below table.

According to Table 7, the average error distance is $28.25 \mathrm{~cm}$, which is much better than the WiFi system.

\section{Healthcare Application}

To combine this system with healthcare application, we must find the demands. It is common for doctors to carry patient's case during daily ward round. So our target is to simply the work of doctors. When users click the healthcare button and switch the localization button on, it will map current self-location and all patients' location. Figure 25 Then doctors can click nearby button to view surrounding patients' list. For instance, when the doctor stand by the patient $\mathrm{A}$ if he is trying to check in the diagnosis of patient A. he can press the "nearby" button and then he can easily find patient A from the nearby list, nevertheless, patient B who is far from the doctor or even in another ward will not be listed in the nearby list. (Figure 26) This will definitely improve the efficiency of doctors' work. Furthermore, this application can also remind doctors which patients have been checked and which one has not. If the doctor has checked the patient, the doctor can mark it. It will not display on the screen until the end of this ward round.

Further more, this application can also develop a sub-application for patients for indoor navigation. To help patients to find right direction and right way, we also use the compass in iPhone, which will help both doctors and patients to find the direction. Especially, for patient, who newly come to a hospital. It is quite difficult for them to find a right place and room to deal with the hospital issues. For instance, for patients, who should take X-ray in diagnostic imaging department. But
Table 7. Test results of ZigBee location system.

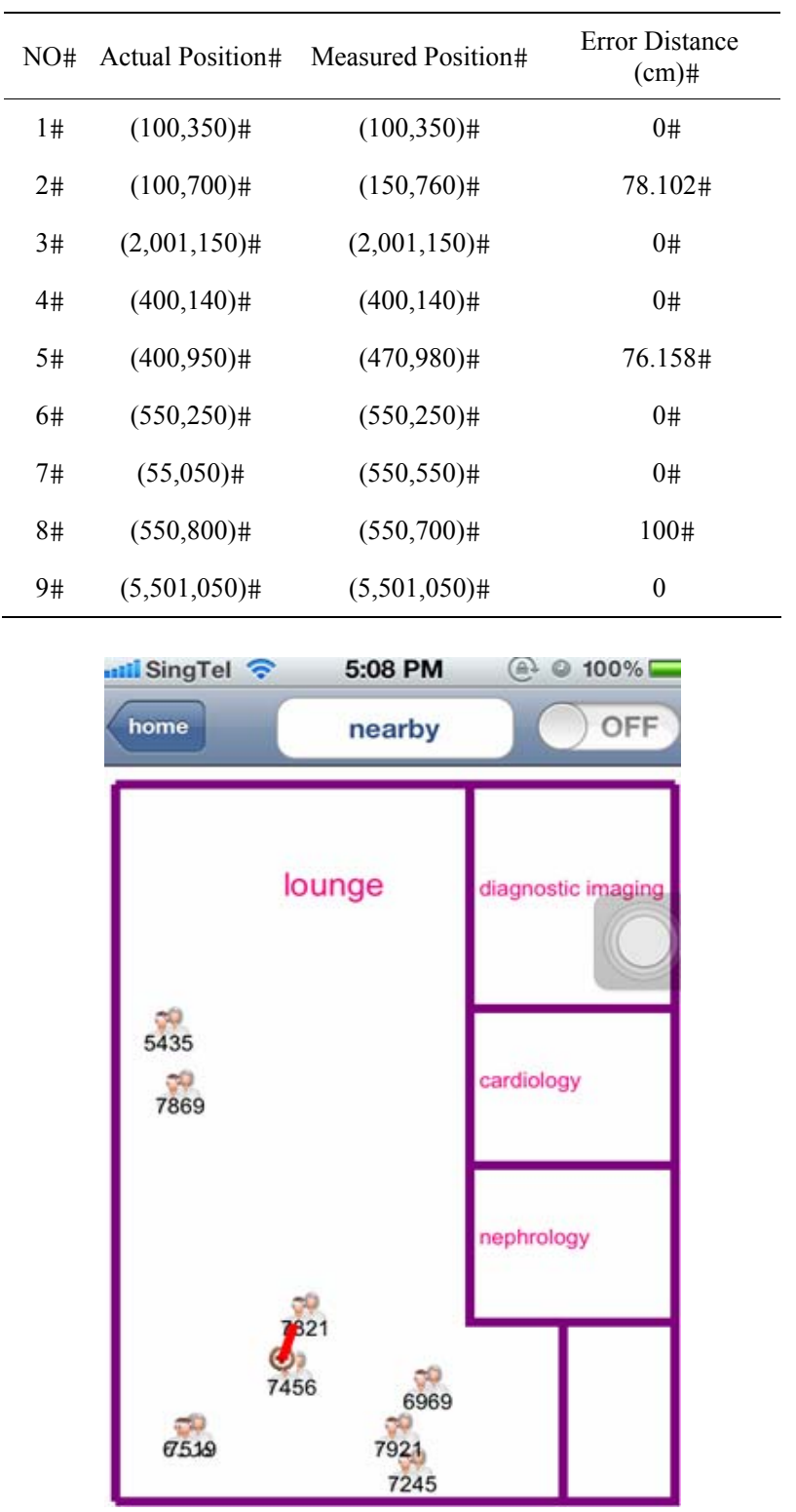

Figure 25. Doctor's application.

there are lots of departments in a hospital and there may be crowded which proves inconvenient for patients. With this application, nevertheless, patients don't have to worry about loss their way in hospitals. It can guide patients to go to right place. As we can see from the picture, the array direction is the direction that the patient is facing.

The other function of this application is to track patients (Figure 27). For mental disease patients, they need to be tracked to prevent accidents and escape from hospital. Hence setting some restricted zone is necessary to alert doctors to their patients' unusual locations and behaviors. In the tracking function, if a patient goes into restricted area, the application will alert. 


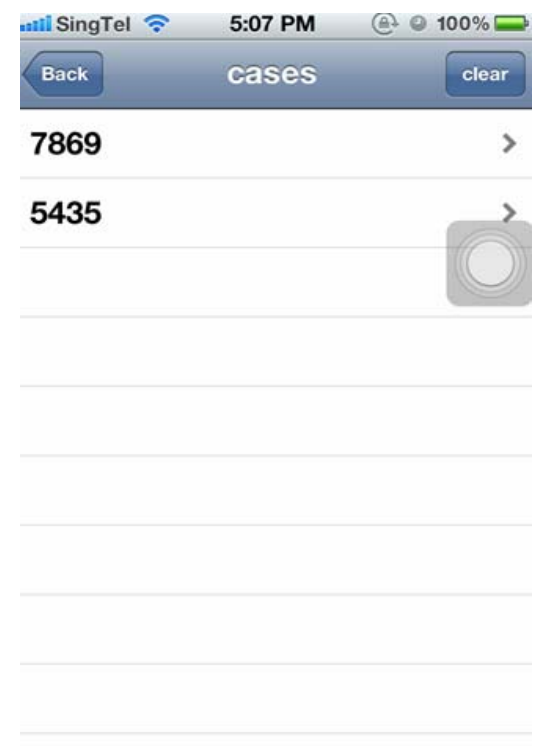

Figure 26. Nearby patient's list.

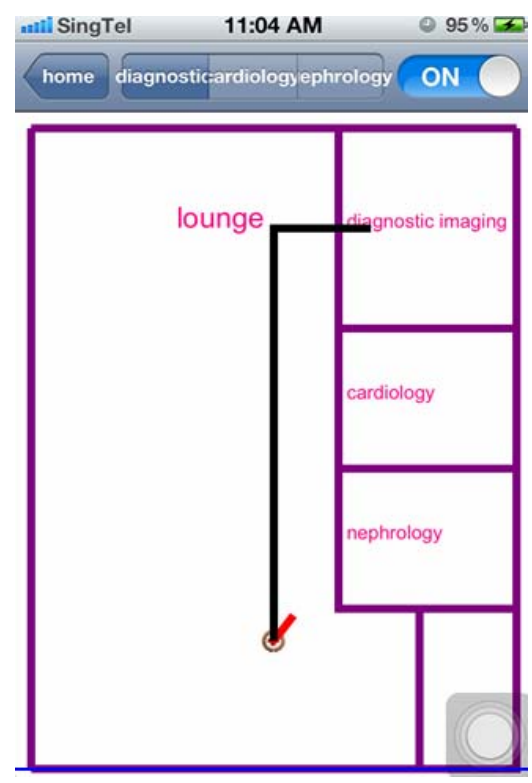

Figure 27. Navigation function.

\section{Conclusion}

To be more practical and efficient, the next step is to use it in hospital to verify its result. Furthermore, to improve the accuracy, adding more APs is an effective way. However, it will increase the time delay during localization, which will decrease the real-time sensitivity. So we should find a balance between the accuracy, sensitivity and complexity of a practical real-time RTLS system.

\section{REFERENCES}

[1] L. M. Ni, Y. H. Liu, Y. C. Lau and A. P. Patil, "LANDMARC: Indoor Location Sensing Using Active RFID," Proceedings of the 1st IEEE International Conference on Pervasive Computing and Communications, March 2003.

[2] J. Wyffels, J.-P. Goemaere, P. Verhoeve, P. Crombez, B. Nauwelaers, L. DeStrycker, K. A. H. O. Sint-Lieven, K. U. Leuven and N. V. Televic, "A Novel Indoor Localization System for Healthcare Environments," International Symposium on Computer-Based Medical Systems (CBMS), 2012.

[3] M. Pourhomayoun, Z. P. Jin and M. Fowler, "Spatial Sparsity Based Indoor Localization in Wireless Sensor Network for Assistive Healthcare," IEEE International Conference on Engineering in Medicine and Biology Society $(E M B C), 2012$.

[4] M. Bharanidharan, X. J. Li, Y. Y. Jin, J. S. Pathmasuntharam and G. X. Xiao, "Design and Implementation of a Real Time Locating System Utilizing WiFi Signals from iPhones," IEEE International Conference on Networks (ICON), 2012.

[5] M. Ali, "iPhone SDK Programming: Developing Mobile Applications for Apple iPhone and iPod Touch," Wiley Press, 2009.

[6] Q. X. Chen; D.-L. Lee and W.-C. Lee, "Rule-Based WiFi Localization Methods," IEEE/IFIP International Conference on Embedded and Ubiquitous Computing, 2008.

[7] T. Bagosi and Z. Baruch, "Indoor Localization by WiFi," IEEE International Conference on Intelligent Computer Communication and Processing (ICCP), 2011.

[8] Effelsberg, "COMPASS: A Probabilistic Indoor Positioning System Based on 802.11 and Digital Compasses," WiNTECH '06 Proceedings of the 1st International Workshop on Wireless Network Testbeds, Experimental Evaluation \& Characterization, 2006, pp. 34-40.

[9] W.-H. Chen, H. H. Chang; T. H. Lin, P. C. Chen, L. K. Chen, S. J. Hwang, D. H. J. Yen, H. S. Yuan and W. C. Chu, "Dynamic Indoor Localization Based on Active RFID for Healthcare Applications: A Shape Constraint Approach," International Conference on Biomedical Engineering and Informatics (BMEI), 2009.

[10] L. Y. Hao, G. Y. Liang and L. Wei, "Indoor Positioning system ( Middleware)," Nanyang Technological University, Nanyang, 2012. 